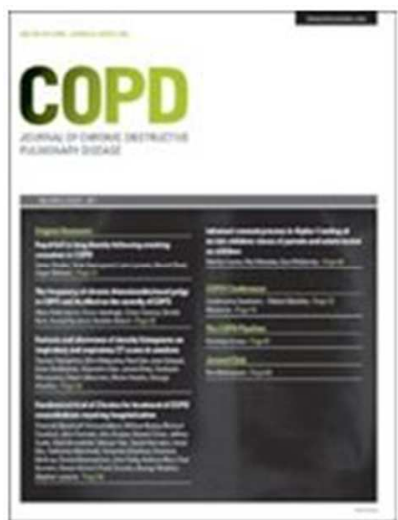

\title{
Computerized respiratory sounds in patients with COPD: a systematic review
}

\begin{tabular}{|r|l|}
\hline Journal: & COPD: Journal Of Chronic Obstructive Pulmonary Disease \\
\hline Manuscript ID: & COPD-2013-0201.R1 \\
\hline Manuscript Type: & Clinical Review \\
\hline Date Submitted by the Author: & n/a \\
\hline Complete List of Authors: & $\begin{array}{l}\text { Jácome, Cristina; University of Aveiro, School of Health Sciences } \\
\text { Marques, Alda; University of Aveiro, School of health Sciences (ESSUA) }\end{array}$ \\
\hline Keywords: & $\begin{array}{l}\text { computerized respiratory sounds, COPD, adventitious respiratory sounds, } \\
\text { normal respiratory sounds }\end{array}$ \\
\hline
\end{tabular}

\section{SCHOLARONE ${ }^{\text {m }}$}

Manuscripts 
Title: Computerized respiratory sounds in patients with COPD: a systematic review Running head: Computerized respiratory sounds in COPD Cristina Jácome ${ }^{1,2}$, Alda Marques ${ }^{2,3}$

1 Research Centre in Physical Activity, Health and Leisure (CIAFEL), Faculty of Sports, University of Porto, Portugal

2 School of Health Sciences, University of Aveiro (ESSUA), Aveiro, Portugal

3 Unidade de Investigação e Formação sobre Adultos e Idosos (UniFAl), Porto, Portugal

Declaration of interest

The authors report no declarations of interest. Support for this study was provided by Fundação para a Ciência e Tecnologia (FCT - Ref. SFRH/BD/84665/2012), Portugal.

Corresponding author: Alda Marques, School of Health Sciences, University of Aveiro (ESSUA), Campus Universitário de Santiago, Edifício III, 3810-193 Aveiro, Portugal. Telephone: 00351 234372 462. Email: amarques@ua.pt

Key-words: computerized respiratory sounds; normal respiratory sounds; adventitious respiratory sounds; chronic obstructive pulmonary disease; crackles; wheezes 
ABSTRACT

Computerized respiratory sound analysis provides objective information about the respiratory system and may be useful to monitor patients with chronic obstructive pulmonary disease (COPD) and detect exacerbations early. For these purposes, a thorough understanding of the typical computerized respiratory sounds in patients with COPD during stable periods is essential. This review aimed to systematize the existing evidence on computerized respiratory sounds in stable COPD.

A literature search in the Medline, EBSCO, Web of Knowledge and Scopus databases was performed.

Seven original articles were included. The maximum frequencies of normal inspiratory sounds at the posterior chest were between 113 and $130 \mathrm{~Hz}$, lower than the frequency found at trachea $(228 \mathrm{~Hz})$. During inspiration, the frequency of normal respiratory sounds was found to be higher than expiration (130 vs. $100 \mathrm{~Hz})$. Crackles were predominantly inspiratory (2.9-5 vs. expiratory $0.73-2)$ and characterized by long durations of the variables initial deflection width $(1.88-2.1 \mathrm{~ms})$ and two cycle duration $(7.7-11.6 \mathrm{~ms})$. Expiratory wheeze rate was higher than inspiratory rate. In patients with COPD normal respiratory sounds seem to follow the pattern observed in healthy people and adventitious respiratory sounds are mainly characterized by inspiratory and coarse crackles and expiratory wheezes. Further research with larger samples and following the Computerized Respiratory Sound Analysis (CORSA) guidelines are needed. 


\section{Background}

Chronic Obstructive Pulmonary Disease (COPD) is an important cause of morbidity and mortality worldwide [1], projected to be the seventh leading cause of years lived with disability by 2030 [2]. The COPD trajectory is usually marked by frequent acute exacerbations [3], that lead to patients' health status deterioration and account for the greatest proportion of the COPD burden on the health care systems $[4,5]$. Therefore, significant research efforts have been dedicated to improve the prevention and early detection of exacerbations.

Auscultation of respiratory sounds is widely used by health professionals for monitoring respiratory diseases [6], such as COPD, as it provides information about the respiratory function and structure that cannot be obtained with any other simple and non-invasive method [7]. However, auscultation with a stethoscope is a subjective process depending on human's ear auditory system and memory capacities [8], terminology used, qualitative nature of respiratory sounds [9] and stethoscope acoustics specifications [10].

Computerized respiratory sound analysis, which consists of recording patients' respiratory sounds with an electronic device and classifying/analyzing them based on specific signal characteristics, overcomes the identified limitations with the standard auscultation $[9,11,12]$. Nevertheless, the implementation of computerized respiratory sound analysis alone is insufficient to improve the diagnostic value of auscultation in monitoring patients with COPD and in detecting COPD exacerbations. Even with an objective method, health professionals cannot interpret with confidence the computerized respiratory sound analysis findings (e.g., presence/absence of an exacerbation), without a clear definition of what are the typical auscultation findings in patients with COPD during stable periods. Thus, this review aimed to systematize the existing evidence on computerized respiratory sounds in stable COPD.

\section{Methods}

Search strategy

An extensive literature search was performed from March to May 2013 in the following electronic databases Medline (1948-2013), EBSCO (1974-2013), Web of Knowledge (19702013) and Scopus (1960-2013) databases. The search terms were based on a combination of the following keywords: [COPD OR "chronic obstructive pulmonary disease" OR "chronic 
bronchitis" OR emphysema] and ["auscultation" OR "digital auscultation" OR "electronic auscultation" OR "computerized analyses" OR "digital signal process"” OR "acoustic signal process ${ }^{* "}$ OR "computerized lung sound analysis" OR "automated classification of lung sounds"] and ["lung sounds" OR "breath sounds" OR "respiratory sounds" OR "Adventitious lung sounds" OR "Adventitious sounds" OR Crackle* OR Wheez ${ }^{*}$. The search terms were limited to titles and abstracts. The reference lists of the selected articles were scanned for other potential eligible studies. This systematic review was reported according to preferred reporting items for systematic reviews and meta-analyses (PRISMA) guidelines [13].

Eligibility criteria

According to the PICO (Population, Intervention, Comparison, and Outcome) framework, studies were included if they met the following inclusion criteria:

i) Population: patients with COPD;

ii) Intervention: none;

iii) Comparison: none;

iv) Outcomes: parameters of computerized respiratory sounds (normal and/or adventitious respiratory sounds).

Articles were also included if i) were full papers published as original articles or in conference proceedings and ii) were written in English, Portuguese, Spanish or French. Articles were excluded when the respiratory sounds were characterized through standard auscultation. Book chapters, review papers, abstracts of communications or meetings, letters to the editor, commentaries to articles, unpublished work and study protocols were also excluded from this review.

Study selection

Duplicates were first removed. Then, the title, abstract and keywords were analyzed to assess the type and relevance of the publication for the scope of the review. If the publication was potentially relevant for the scope of the review, the full-text was screened for content to decide their inclusion. The two reviewers decided the articles inclusion and disagreements were solved by consensus.

Data extraction 
Data from the included articles were extracted in a structured table-format, i.e.: first author's last name and year of publication, study design, participants, data collection protocol, data analyses, outcomes and quantitative findings.

Quality assessment

The quality of the included studies was assessed with the 'Crombie criteria' for assessment of cross-sectional studies $[14,15]$. The 'Crombie criteria' assesses mainly the research design, the sample recruitment and representativeness, the reliability of the measurements and the statistical analysis. The quality of each study was assessed independently by the two reviewers and when disagreements occurred, consensus was achieved through discussion.

Data analysis

To determine the consistency of the quality assessment performed by the two reviewers, an inter-observer agreement analysis using the Cohen's kappa was performed. The value of Cohen's kappa ranges from 0 to 1 and can be categorized as slight (0.0-0.20), fair (0.21-0.40), moderate $(0.41-0.60)$, substantial $(0.61-0.80)$ or almost perfect $(\geq 0.81)$ agreement [16]. This statistical analysis was performed using IBM SPSS Statistics version 20.0 (IBM Corporation, Armonk, NY, USA).

\section{Results}

Study selection

The database search identified 68 records. After duplicates removal, 60 records were screened for relevant content. During the title, abstract and keyword screening, 46 articles were excluded. The full-text of the 14 potentially relevant articles was assessed and 8 articles were excluded due to the following reasons: use of standard auscultation to characterize respiratory sounds $(n=4)$, detection of adventitious respiratory sounds through imaging techniques $(n=3)$ and results from patients with COPD were not individualized $(n=1)$. Six original articles were selected. The search for relevant articles within the reference list of the selected articles retrieved 1 study which was also included. Therefore, 7 original articles were included in this review.

(insert figure 1 about here)

Quality assessment 
The quality of the included studies, using the 'Crombie criteria', is presented in table 1 . All studies included had an appropriate research design and used objective measures. Two studies failed in reporting the recruitment strategy used [17, 18]. As no study reported dropouts, the response rate indicator was considered in all studies. All presented the statistical analyses used, with one exception [18], which were appropriate. Studies did not use representative samples or justified their size. Evidence of bias was not considered present, despite the use of convenience samples. The agreement between the two reviewers was substantial $(k=0.714$; $95 \% \mathrm{Cl}$ 0.532-0.892; $\mathrm{p}=0.001)$.

(insert table 1 about here)

Study characteristics

A total of 164 patients with stable COPD participated in the included studies. All studies, with one exception [18], provided data regarding patients' mean age, which ranged from 46 to 66.3 years old. Patients' mean forced expiratory volume in 1 second $\left(\mathrm{FEV}_{1}\right)$ ranged between 36 and $54.5 \%$ of the predicted $[17,19-21]$.

The protocols used to record the respiratory sounds were different in all studies. Piirila et al. (1991) [17] reported that respiratory recordings were obtained with the patient in the sitting position. The other authors were not clear about the patients' body position during the recordings. Respiratory sounds were recorded while patients breathed with an airflow between 1 and $1.5 \mathrm{~L} / \mathrm{s}[17,19,21]$ and during forced expiratory maneuvers [20]. However, some studies did not report the respiratory maneuvers used during the respiratory sounds recordings [18, 22, 23].

Respiratory sounds were recorded with microphones (condenser [19], electret condenser [2123] and miniature electret [18]) and piezoelectric contact sensors [19, 20]. Two studies recorded respiratory sounds only at one chest location: at trachea [20] and at the base of the right posterior chest [22]. However, the majority of studies recorded respiratory sounds in more than one chest location: i) at chest sites with abnormal sounds [23]; ii) at trachea and at the base of the right posterior chest [19]; iii) at posterior right/left chest [17]; iv) at trachea, right/left axillae and right/left posterior bases [21]; and trachea, lateral bases and posterior chest [18]. 
Regarding pre-processing methods, five studies reported the methods used to filter the respiratory sounds signals. In two studies, high- and low-pass filters were used, with cut-off frequencies from $50-100 \mathrm{~Hz}$ and from $4,000-5,000 \mathrm{~Hz}[17,19]$. Three studies, instead, used band-pass filters $(80-2,000 \mathrm{~Hz}[20,23]$ and $60-2,100 \mathrm{~Hz}[21])$. In relation to digitization protocols, five studies described the sampling rates used, which ranged from $5,000 \mathrm{~Hz}$ to $20,000 \mathrm{~Hz}[17,19-22]$.

The characteristics of the respiratory sounds were mainly explored using frequency analyses [17, 19-22]. Fast Fourier Transform (FFT) analysis was used in four studies, one study used FFT alone [19], two combined FFT with time-expanded waveform analysis [17, 22] and one combined FFT with algorithms [20]. Time-expanded waveform analysis alone [23], a timefrequency wheeze detector [21] and an algorithm that automatically analyzed acoustic energy versus time [18] were also used.

Synthesis of the results

The results were summarized in two categories: normal respiratory sounds and adventitious respiratory sounds. Detailed information about each study is provided in table 2.

(insert table 2 about here)

Normal respiratory sounds

Two studies characterized normal respiratory sounds of patients with COPD, by breathing phase [17] and only in the inspiratory phase [19]. Similar maximum frequencies of normal inspiratory sounds acquired at the posterior chest wall, $130 \mathrm{~Hz}$ [17] and $113 \mathrm{~Hz}$ [19], were reported. The total power spectra, maximum frequency, upper frequency limits for the 2nd and 3rd quartiles of the power spectra were higher in the respiratory sounds recorded at trachea than posterior chest [19]. It was also showed that the maximum frequency and upper frequency at $-20 \mathrm{~dB}$ were higher in inspiratory than expiratory respiratory sounds [17].

\section{Adventitious respiratory sounds}

Six of the included studies analyzed the characteristics of adventitious respiratory sounds: crackles [17, 18, 22, 23], wheezes [18, 20, 21] and rhonchi [18].

\section{Crackles}


The characteristics of inspiratory and expiratory crackles were explored by two studies $[17,18]$. Munakata et al. [22] only looked at inspiratory crackles and Bettencourt et al.[23] did not differentiate between inspiratory and expiratory crackles. Inspiratory crackles (between 2.9 and 5) were more frequent than expiratory (between 0.73 and 2)[17, 18]. The variable initial deflection width (IDW) was found to be between 1.88 and $2.1 \mathrm{~ms}$ and the variable two cycle duration (2CD) between 7.74 and $11.6 \pm 1.1 \mathrm{~ms}[17,22]$. Shorter durations, IDW $0.91 \mathrm{~ms}$ and $2 \mathrm{CD}$ $5.4 \mathrm{~ms}$, were however also reported [23]. The peak frequency of inspiratory crackles was found to be $233 \mathrm{~Hz}$ and the maximum frequency $394 \mathrm{~Hz}$ [22]. Piirila et al. also studied the direction of the crackles first deflection and verified that during inspiration the majority were downward $(90 \%$ vs. $10 \%$ upward) and during expiration were relatively similar (upward $47 \%$ vs. downward $53 \%$ ) [17].

\section{Wheezes}

The three studies that analyzed the characteristics of wheezes used different protocols to record the respiratory sounds and different recording devices. The number of wheezes identified during 5 minutes of normal breathing was on average 42 [21] and during forced expiratory maneuvers 10.4 [20]. During forced expiratory maneuvers, only $13.7 \%$ of the time was not occupied by wheezes, and most wheezes were polyphonic $(53.6 \%$ vs. $32.6 \%$ monophonic) [20]. Their mean frequency of the originated wheezes was $669.4 \mathrm{~Hz}$ [20]. Wheezes were found to be more frequent during expiration than in inspiration (inspiratory wheeze rate $2 \%$ vs. expiratory wheeze rate 12\%) [18].

\section{Rhonchi}

Expiratory rhonchi rate in patients with COPD was found to be higher than the inspiratory rate (7\% vs. $3 \%)[18]$.

\section{Discussion}

The major findings of this systematic review were that i) normal respiratory sounds of patients with COPD follow the pattern observed in healthy people and ii) adventitious respiratory sounds are mainly characterized by inspiratory and coarse crackles and expiratory wheezes.

In patients with COPD, the maximum frequencies of normal inspiratory sounds at the posterior chest were between 113 [19] and $130 \mathrm{~Hz}$ [17], recorded at $1 \mathrm{~L} / \mathrm{s}$ [17] and at 1-1.25L/s [19]. In a 
group of healthy people, Malmberg et al. (1995) found similar maximum frequencies $(117 \mathrm{~Hz})$ [19]. Therefore, as pointed out by Scheur et al. (1992) and Malmberg et al. (1995), the frequency and intensity of normal respiratory sounds in patients with COPD are similar to those found in healthy people $[19,24]$. The frequency of normal respiratory sounds was found to be higher during inspiration than expiration [17]. This finding is in line with previous literature describing the normal respiratory sounds of healthy people [25] and of people with chronic diseases, such as bronchiectasis, fibrosing alveolitis and asbestos-related pleural disease [17, 26]. Normal respiratory sounds at the trachea presented higher frequencies than sounds at the posterior chest. This difference has been explained by the specific characteristics of these chest locations. At trachea turbulent flows are generated, due to its large diameter and absence of a filter [27, 28]. Conversely, at posterior chest the flow becomes laminar and the high frequencies are filtered by the parenchyma $[27,28]$.

In patients with COPD, crackles were more common during inspiration (between 2.9 and 5 [17, 18]) than during expiration (between 0.73 and $2[17,18]$ ). These data is in accordance with the Computerized Respiratory Sound Analysis (CORSA) definition of crackles, "adventitious, discontinuous, explosive sound occurring usually during inspiration" [29]. In healthy people, this crackling behavior is also verified, however, with fewer crackles identified in each breathing phase (inspiration $1 \pm 2$ vs. expiration $1 \pm 1$ ) [18]. In inspiratory crackles, the IDW was found to be between 1.88 and $2.1 \mathrm{~ms}[17,22]$ and the $2 \mathrm{CD}$ between 7.74 and $11.6 \pm 1.1 \mathrm{~ms}[17,22]$. According to the CORSA, these time parameters are characteristic of coarse crackles, defined as "low pitched and with a high amplitude and long duration" [29]. Bettencourt et al.[23], in a group of patients with COPD, reported shorter durations of the IDW $(0.91 \mathrm{~ms})$ and of the $2 \mathrm{CD}$ (5.4ms). However, as in this study the beginning of the crackle was manually annotated, these shorter durations may be explained by the known difficulty in determine the exact beginning of a crackle [30]. Another reason that could explain these results was the inclusion of patients with different disease severities, however, this is unknown as studies failed in characterizing patients' COPD grade and only Piirila et al. provided the values of the $\mathrm{FEV}_{1} \%$ predicted. In patients with idiopathic pulmonary fibrosis, bronchiectasis, pneumonia and fibrosing alveolitis shorter durations of IDW and 2CD have been found [17, 22, 31]. 
Only three studies analyzed the characteristics of wheezes and all used different protocols to record the respiratory sounds $[18,20,21]$, which limited the synthesis of the results. Only one study analyzed the presence of wheezes in patients with COPD during normal breathing and found an average of 42 wheezes recorded during 5 minutes [21]. However, this study assessed a convenience sample of 7 patients, which already presented wheezes during standard auscultation, and therefore, this number of wheezes may not be typical in all patients with COPD. Murphy verified that wheezes were more frequent during expiration than in inspiration $(12 \%$ vs. $2 \%)$ [18]. This is in line with the wheezes pattern found in healthy people, in patients with asthma, congestive heart failure and pneumonia [18]. During forced expiratory maneuvers, $86.3 \%$ of the time was occupied by wheezes, and the greatest part of wheezes generated were polyphonic [20]. Conversely, in patients with asthma, the majority of wheezes identified were monophonic and a lower wheeze rate was found (77.9\%) [20]. This result was expected as wheezes are produced by fluttering of the airways and COPD is more associated with a reduction on bronchial stiffness than asthma [32].

Expiratory rhonchi rate in patients with COPD was higher than the inspiratory rate ( $7 \%$ vs. $3 \%)$ [18]. This was expected since this adventitious respiratory sound is a low-pitched wheeze [33]. In healthy people, rhonchi are almost absent (average rate in inspiration $0 \pm 1$ and expiration $0 \pm 3)[18]$.

This systematic review has important limitations that need to be considered. The literature search was performed in four electronic databases (Medline, EBSCO, Web of Knowledge and Scopus). However, other electronic databases, such as the IEEE (Institute of Electrical and Electronics Engineers) Xplore, which is a resource for electrical engineering and computer science publications, were not used and thus other articles may have been missed. Nevertheless, as the search strategy was thorough and further complemented with the review of reference lists from the articles included, it is believed that this review contains the most relevant studies on the topic analyzed. The included studies met only $4 / 5$ quality indicators from the 8 assessed in the Crombie criteria, indicating low/medium methodological quality. However, strict criteria for study methodological quality have only become common practice in recent years and most studies were published before 2000. Nonetheless, it is believed that the 
inclusion of these studies in this review provided valuable insights into respiratory sounds characteristics in COPD.

Only seven studies with small sample sizes were included demonstrating that the available evidence about computerized respiratory sounds in patients with COPD is still limited. Samples were mainly composed of young-old patients and with advanced disease. Therefore, the extent to which the conclusions of this review are also applicable to oldest-old patients with COPD or with early COPD remains unclear. Furthermore, in the studies analyzed, respiratory sounds characteristics have not been compared across different patients with COPD (e.g., age, gender, disease severity, smoking history, etc.), thus conclusions regarding the existence of different phenotypes on respiratory sounds could not be drawn. In a recent study with patients with acute exacerbations of COPD, it was possible to characterize the course of exacerbations into two phenotypes based on the variation of specific respiratory sound characteristics [34]. Future research should clarify if different phenotypes exist during stable phases or if they become evident only during exacerbation periods. FFT was used to analyze respiratory sounds in most studies. However, as respiratory sounds are non-stationary signals, conventional methods of frequency analysis may not be recommended [34]. Instead, short-time fourier transform should be considered to characterize respiratory sounds in future studies [33].

A lack of standardization across all studies in the procedures used to record (patient' body position, respiratory maneuvers, chest locations, sensor type), analyze (filters, sampling rates, FFT, algorithms) and characterize (parameters selected) respiratory sounds was found. In a recent systematic review on respiratory sounds in healthy people, these methodological differences were also observed [35]. Guidelines for research and clinical practice in the field of respiratory sounds have been published in 2000 by the CORSA project group [33]. These guidelines standardized the instrumentation, ways of acquiring data, procedures and signal processing techniques as well as the respiratory sounds' nomenclature [33]. Therefore, the inconsistence of the procedures was expected in studies conducted in the 90s, however, not in the three studies published after 2000. This lack of standardization made interpretation and synthesis of the results difficult. Future studies in the field of respiratory sounds should follow the CORSA guidelines. 
The overall findings of this review, together with findings from future studies using advanced auscultation equipment and analysis methods, will establish the characteristics of respiratory sounds in patients with COPD. Since this relevant information can be obtained with a noninvasive and cost-effective method, the potential of computerized respiratory sounds to monitor patients' respiratory status, e.g., in telemedicine applications, has become evident.

\section{Conclusion}

In patients with COPD normal respiratory sounds seem to follow the pattern observed in healthy people and adventitious respiratory sounds are mainly characterized by inspiratory and coarse crackles and expiratory wheezes. However, these conclusions were drawn based in few studies conducted with small sample sizes of patients with advanced COPD and presenting a high inconsistence among the procedures used. Further research with larger samples, incorporating patients with different age ranges and with all COPD grades, and following the CORSA guidelines are needed to define the characteristics of computerized respiratory sounds in patients with COPD. 


\section{Acknowledgements \\ CJ is supported by Fundação para a Ciência e Tecnologia (PhD grant - Ref. SFRH/BD/84665/2012), Portugal. \\ References}

1. Mannino DM, Braman S. The epidemiology and economics of chronic obstructive pulmonary disease. Proc Am Thorac Soc. 2007;4(7):502-6.

2. Mathers CD, Loncar D. Projections of global mortality and burden of disease from 2002 to 2030. PLoS Med. 2006;3(11):e442.

3. Vestbo J, Hurd SS, Agustí AG, Jones PW, Vogelmeier C, Anzueto A, et al. Global Strategy for the Diagnosis, Management, and Prevention of Chronic Obstructive Pulmonary Disease. Am J Respir Crit Care Med. 2013;187(4):347-65.

4. Anzueto A. Impact of exacerbations on COPD. Eur Respir Rev. 2010;19(116):113-8.

5. Seemungal TA, Hurst JR, Wedzicha JA. Exacerbation rate, health status and mortality in COPD: a review of potential interventions. Int J Chron Obstruct Pulmon Dis. 2009;4:203-23.

6. Marques A, Bruton A, Barney A. Clinically useful outcome measures for physiotherapy airway clearance techniques: a review. Physical Therapy Reviews. 2006;11(4):299-307.

7. $\quad$ Forgacs P. Lung sounds. London: Bailliere Tindall; 1978.

8. Welsby PD, Parry G, Smith D. The stethoscope: some preliminary investigations. Postgrad Med J. 2003;79(938):695-8.

9. Polat H, Guler I. A simple computer-based measurement and analysis system of pulmonary auscultation sounds. J Med Syst. 2004;28(6):665-72.

10. Welsby PD, Earis JE. Some high pitched thoughts on chest examination. Postgrad Med J. 2001;77(912):617-20.

11. Moussavi ZK, Leopando MT, Pasterkamp H, Rempel G. Computerized acoustical respiratory phase detection without airflow measurement. Med Biol Eng Comput. 2000;38(2):198-203.

12. Kandaswamy A, Kumar CS, Ramanathan RP, Jayaraman S, Malmurugan N. Neural classification of lung sounds using wavelet coefficients. Comput Biol Med. 2004;34(6):523-37. 
13. Moher D, Liberati A, Tetzlaff J, Altman DG, on behalf of the PRISMA group. Preferred reporting items for systematic reviews and meta-analyses: The PRISMA statement. Ann Intern Med. 2009;151(4):264-9.

14. Crombie I. The pocket guide to critical appraisal. London: BMJ Publishing Group; 1996.

15. Petticrew M, Roberts $\mathrm{H}$. Systematic reviews in the social sciences: a practical guide. Oxford: Blackwell; 2006.

16. Landis JR, Koch GG. The Measurement of Observer Agreement for Categorical Data. Biometrics. 1977;33(1):159-74.

17. Piirila P, Sovijarvi AR, Kaisla T, Rajala HM, Katila T. Crackles in patients with fibrosing alveolitis, bronchiectasis, COPD, and heart failure. Chest. 1991;99(5):1076-83.

18. Murphy RLH, Jr. Special articles: in defense of the stethoscope. Respir Care. 2008;53(3):355-69.

19. Malmberg LP, Pesu L, Sovijarvi AR. Significant differences in flow standardised breath sound spectra in patients with chronic obstructive pulmonary disease, stable asthma, and healthy lungs. Thorax. 1995;50(12):1285-91.

20. Fiz JA, Jané R, Homs A, Izquierdo J, García MA, Morera J. Detection of wheezing during maximal forced exhalation in patients with obstructed airways. Chest. 2002;122(1):18691.

21. Taplidou SA, Hadjileontiadis LJ. Wheeze detection based on time-frequency analysis of breath sounds. Comput Biol Med. 2007;37(8):1073-83.

22. Munakata M, Ukita H, Doi I, Ohtsuka Y, Masaki Y, Homma Y, et al. Spectral and waveform characteristics of fine and coarse crackles. Thorax. 1991;46(9):651-7.

23. Bettencourt PE, Delbono EA, Spiegelman D, Hertzmark E, Murphy RLH. Clinical utility of chest auscultation in common pulmonary-diseases. Am J Respir Crit Care Med. 1994;150(5):1291-7.

24. Schreur HJW, Sterk PJ, Vanderschoot J, Vanklink HCJ, Vanvollenhoven E. Lung sound intensity in patients with emphysema and in normal subjects at standardized air-flows. Thorax. 1992;47(9):674-9. 
25. Kompis M, Pasterkamp H, Oh Y, Wodicka GR. Distribution of inspiratory and expiratory respiratory sound intensity on the surface of the human thorax. 19th Annual International Conference of the IEEE; Chicago, IL1997. p. 2047-50.

26. Piirila $\mathrm{P}$, Lehtola $\mathrm{H}$, Zitting A, Kivisaari L, Koskinen $\mathrm{H}$, Luukkonen R, et al. Lung sounds in asbestos induced pulmonary disorders. Eur Respir J. 2000;16(5):901-8.

27. Bohadana $\mathrm{AB}$. Lung sounds in asthma and chronic obstructive pulmonary disease. Monaldi Arch Chest Dis. 2000;55(6):484-7.

28. Sovijärvi ARA, Malmberg LP, Charbonneau G, Vanderschoot J, Dalmasso F, Sacco C, et al. Characteristics of breath sounds and adventitious respiratory sounds. Eur Respir Rev. 2000;77(10):591-6.

29. Sovijärvi ARA, Dalmasso F, Vanderschoot J, Malmberg LP, Righini G, Stoneman SAT. Definition of terms for applications of respiratory sounds. Eur Respir Rev. 2000;77(10):597-610.

30. Piirila P, Sovijarvi A. Crackles: recording, analysis and clinical significance. Eur Respir J. 1995;8(12):2139-48.

31. Ponte DF, Moraes R, Hizume DC, Alencar AM. Characterization of crackles from patients with fibrosis, heart failure and pneumonia. Med Eng Phys. 2013;35(4):448-56.

32. Meslier N, Charbonneau G, Racineux JL. Wheezes. Eur Respir J. 1995;8(11):1942-8.

33. Sovijarvi ARA, Vanderschoot J, Earis JE. Standardization of computerized respiratory sound analysis. Eur Respir Rev. 2000;10(77):585.

34. Sánchez Morillo D, Astorga Moreno S, Fernández Granero MÁ, León Jiménez A. Computerized analysis of respiratory sounds during COPD exacerbations. Comput Biol Med. 2013;43(7):914-21.

35. Oliveira A, Marques A. Respiratory sounds in healthy people: a systematic review. Respir Med. 2014(Jan 21 [Epub ahead of print]). 


\section{Figure captions}

Fig. 1 - PRISMA flow diagram of the included studies 
Table 1 - Quality assessment based on the 'Crombie criteria'.

\begin{tabular}{|c|c|c|c|c|c|c|c|c|c|}
\hline $\begin{array}{l}\text { Author } \\
\text { (year) }\end{array}$ & $\begin{array}{l}\text { Appropriate } \\
\text { Research } \\
\text { Design }\end{array}$ & $\begin{array}{l}\text { Appropriate } \\
\text { Recruitment } \\
\text { Strategy }\end{array}$ & $\begin{array}{l}\text { Response } \\
\text { Rate }\end{array}$ & $\begin{array}{l}\text { Sample } \\
\text { Representa- } \\
\text { tiveness }\end{array}$ & $\begin{array}{l}\text { Objective } \\
\text { and Reliable } \\
\text { Measures }\end{array}$ & $\begin{array}{l}\text { Power } \\
\text { Calculation/ } \\
\text { Justification } \\
\text { of Numbers }\end{array}$ & $\begin{array}{l}\text { Appropriate } \\
\text { Statistical } \\
\text { Analysis }\end{array}$ & $\begin{array}{l}\text { Evidence of } \\
\text { Bias }\end{array}$ & $\begin{array}{l}\text { Quality } \\
\text { Indicators } \\
\text { Met }\end{array}$ \\
\hline $\begin{array}{l}\text { Piirila et al. } \\
\text { (1991) }\end{array}$ & 4 & & 4 & & 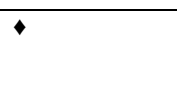 & & 4 & & $4 / 8$ \\
\hline $\begin{array}{l}\text { Munakata et al. } \\
\text { (1991) }\end{array}$ & - & $\bullet$ & - & & - & & • & & $5 / 8$ \\
\hline $\begin{array}{l}\text { Bettencourt et al. } \\
\text { (1994) }\end{array}$ & - & $\bullet$ & $\bullet$ & & - & & • & & $5 / 8$ \\
\hline $\begin{array}{l}\text { Malmberg et al. } \\
\text { (1995) }\end{array}$ & $\bullet$ & $\bullet$ & - & & - & & $\bullet$ & & $5 / 8$ \\
\hline $\begin{array}{l}\text { Fiz et al. } \\
(2002)\end{array}$ & $\bullet$ & - & - & & $\bullet$ & & - & & $5 / 8$ \\
\hline $\begin{array}{l}\text { Taplidou et al. } \\
\text { (2007) }\end{array}$ & - & $\bullet$ & - & & - & & • & & $5 / 8$ \\
\hline $\begin{array}{l}\text { Murphy } \\
(2008)\end{array}$ & $\bullet$ & & $\bullet$ & & $\bullet$ & & $\bullet$ & & $4 / 8$ \\
\hline
\end{tabular}


Table 2 - Computerized respiratory sounds in patients with COPD.

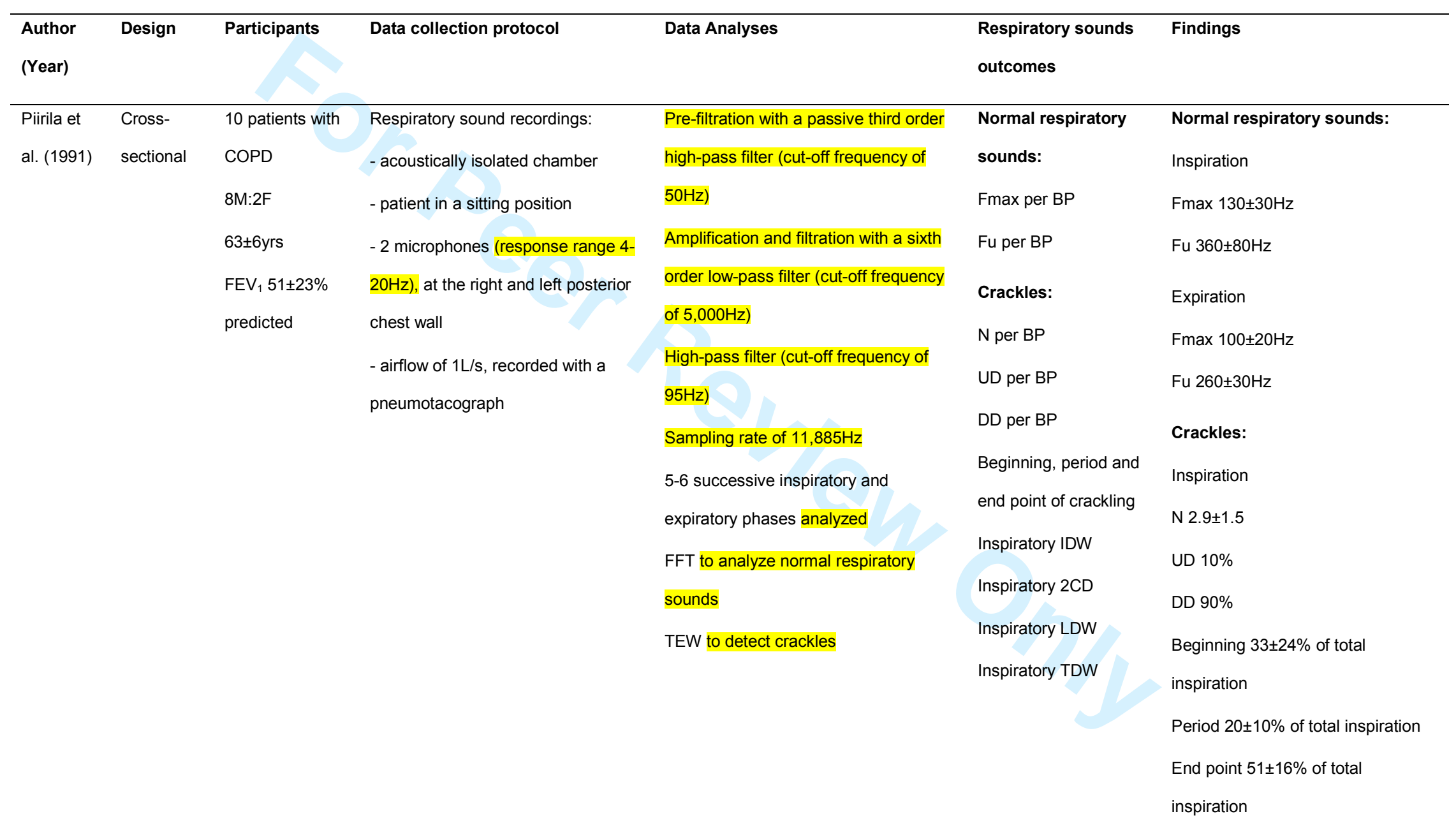


Expiration

N $0.73 \pm 1.14$

UD $47 \%$

DD 53\%

\begin{tabular}{|c|c|c|c|c|c|c|}
\hline Munakata & Cross- & 10 patients with & Respiratory sound recordings: & Sampling rate of $20,000 \mathrm{~Hz}$ & Inspiratory crackles: & Inspiratory crackles: \\
\hline $\begin{array}{l}\text { et al. } \\
\text { (1991) }\end{array}$ & sectional & $\begin{array}{l}\text { COPD } \\
46.0 \pm 10.8 \mathrm{yrs}\end{array}$ & $\begin{array}{l}-1 \text { electret condenser microphone at } \\
\text { the base of the right posterior chest } \\
\text { wall }\end{array}$ & $\begin{array}{l}5 \text { crackles from one inspiratory phase } \\
\text { analyzed } \\
\text { TEW to detect crackles } \\
\text { FFT with a Hanning window for } \\
\text { crackles' frequency analysis } \\
\text { Extraction of the single waveform } \\
\text { signal by cutting at two zero points, } \\
\text { before and after the waveform, and } \\
\text { inserted into a continuous zero } \\
\text { baseline to eliminate background } \\
\text { noises }\end{array}$ & $\begin{array}{l}\text { IDW } \\
1 / 4 C D \\
9 / 4 C D \\
2 C D \\
\text { Fmax } \\
\text { Fpeak }\end{array}$ & $\begin{array}{l}\text { IDW } 1.88 \pm 0.05 \mathrm{~ms} \\
1 / 4 \mathrm{CD} 1.16 \pm 0.03 \mathrm{~ms} \\
9 / 4 \mathrm{CD} 8.79 \pm 0.38 \mathrm{~ms} \\
2 \mathrm{CD} 7.74 \pm 0.32 \mathrm{~ms} \\
\text { Fmax } 394 \pm 10 \mathrm{~Hz} \\
\text { Fpeak } 233 \pm 8 \mathrm{~Hz}\end{array}$ \\
\hline Bettencou & Cross- & 20 patients with & Respiratory sound recordings: & 2 breaths at $2-4$ sites & Crackles: & Crackles: \\
\hline
\end{tabular}




\begin{tabular}{|c|c|c|c|c|c|c|}
\hline $\begin{array}{l}\text { rt et al. } \\
\text { (1994) }\end{array}$ & sectional & $\begin{array}{l}\text { COPD } \\
9 \mathrm{M}: 11 \mathrm{~F} \\
62 \pm 9 \mathrm{yrs}\end{array}$ & $\begin{array}{l}\text { - electret condenser microphone } \\
\text { (connected to the diaphragm of a } \\
\text { stethoscope chest piece) over chest } \\
\text { sites with adventitious respiratory } \\
\text { sounds }\end{array}$ & $\begin{array}{l}\text { Band-pass filter } 80-2,000 \mathrm{~Hz} \\
\text { TEW to detect crackles }\end{array}$ & $\begin{array}{l}\text { IDW } \\
2 \mathrm{CD} \\
\text { ZXS }\end{array}$ & $\begin{array}{l}\text { IDW } 0.91 \pm 0.43 \mathrm{~ms} \\
2 \mathrm{CD} 5.4 \pm 2.4 \mathrm{~ms} \\
\text { ZXS } 4.4 \pm 2.1\end{array}$ \\
\hline $\begin{array}{l}\text { Malmberg } \\
\text { et al. } \\
\text { (1995) }\end{array}$ & $\begin{array}{l}\text { Cross- } \\
\text { sectional }\end{array}$ & $\begin{array}{l}17 \text { patients with } \\
\text { COPD } \\
58(38-73) y r s \\
\text { FEV }_{1} 36(16- \\
79) \% \text { predicted }\end{array}$ & $\begin{array}{l}\text { Respiratory sound recordings: } \\
\text { - sitting position } \\
\text { - } 1 \text { condenser microphone (free field } \\
\text { frequency response } 3-20,000 \mathrm{~Hz} \text { (- } \\
3 \mathrm{~dB} \text { )) at the base of the right } \\
\text { posterior chest wall, approximately } \\
10 \mathrm{~cm} \text { below the margin of the } \\
\text { scapula and } 15 \mathrm{~cm} \text { to the right of the } \\
\text { spine } \\
-1 \text { piezoelectric contact sensor (free } \\
\text { field frequency response essentially } \\
\text { flat ( } \pm 3 \mathrm{~dB} \text { ) within } 100-1,500 \mathrm{~Hz} \text { ) at } \\
\text { the trachea on the right side of the } \\
\text { cricothyroid cartilage } \\
- \text { airflow of } 1-1.25 \mathrm{~L} / \mathrm{s}, \text { recorded with } \\
\text { a pneumotacograph }\end{array}$ & $\begin{array}{l}\text { Pre-filtration with a third order high- } \\
\text { pass filter (cut-off frequency of } 50 \mathrm{~Hz} \text { ) } \\
\text { Amplifier with a flat }( \pm 0-5 \mathrm{~dB} \text { ) frequency } \\
\text { response curve over } 20-20,000 \mathrm{~Hz} \\
\text { Sampling rate of } 12,000 \mathrm{~Hz} \\
\text { Low-pass filter (cut-off frequency of } \\
4,00 \mathrm{~Hz} \text { ) } \\
\text { High-pass filter (cut-off frequency of } \\
100 \mathrm{~Hz} \text { ) } \\
\text { FFT with a Hanning window to analyze } \\
\text { normal respiratory sounds }\end{array}$ & $\begin{array}{l}\text { Inspiratory normal } \\
\text { respiratory sounds: } \\
\text { RMS } \\
\text { Fmax } \\
\text { F50 } \\
\text { F75 }\end{array}$ & $\begin{array}{l}\text { Inspiratory normal respiratory } \\
\text { sounds: } \\
\text { Chest } \\
\text { RMS } 63.5 \pm 4.4 \mathrm{~dB} \\
\text { Fmax } 113 \pm 17 \mathrm{~Hz} \\
\text { F50 } 201 \pm 21 \mathrm{~Hz} \\
\text { F75 } 321 \pm 51 \mathrm{~Hz} \\
\text { Trachea } \\
\text { RMS } 82.6 \pm 3.1 \mathrm{~dB} \\
\text { Fmax } 228 \pm 340 \mathrm{~Hz} \\
\text { F50 } 753 \pm 177 \mathrm{~Hz} \\
\text { F75 } 1239 \pm 186 \mathrm{~Hz}\end{array}$ \\
\hline Fiz et al. & Quasi- & 6 patients with & Respiratory sound recordings: & Amplification and band-pass filter $80-$ & Wheezes: & Wheezes: \\
\hline
\end{tabular}




\begin{tabular}{|c|c|c|c|c|c|c|}
\hline (2002) & $\begin{array}{l}\text { experimen } \\
\text { tal }\end{array}$ & $\begin{array}{l}\text { COPD } \\
6 \mathrm{M}: 0 \mathrm{~F} \\
58.8 \pm 4.9 \mathrm{yrs} \\
\mathrm{FEV}_{1} \\
40.4 \pm 11.9 \% \\
\text { predicted }\end{array}$ & $\begin{array}{l}\text { - } 1 \text { contact microphone }(\mathrm{PPG} \\
\text { sensor, flat response } 50-1,800 \mathrm{~Hz} \text {, } \\
\text { resonance frequency of } 2,600 \mathrm{~Hz}) \text { at } \\
\text { the trachea at the level of the cricoid } \\
\text { cartilage } \\
\text { - during forced expiratory } \\
\text { maneuvers, after deep inspirations } \\
\text { - airflow recorded with a } \\
\text { pneumotachograph }\end{array}$ & $\begin{array}{l}2,000 \mathrm{~Hz} \\
\text { Sampling rate of } 5,000 \mathrm{~Hz} \\
\text { Mean of } 3 \text { forced expiratory maneuvers } \\
\text { analyzed } \\
\text { Modified version of the Shabtai-Musih } \\
\text { et al. algorithm to detect airflow } \\
\text { between } 0.2-1.2 \mathrm{~L} / \mathrm{s} \text { and analyze sound } \\
\text { signal segments of } 128 \text { points } \\
\text { FFT with a Hanning window } \\
\text { Wheeze-grouping algorithm to detect } \\
\text { peaks located in a time-frequency } \\
\text { space }\end{array}$ & $\begin{array}{l}\text { Polyphonic W\% } \\
\text { Time without wheezes }\end{array}$ & $\begin{array}{l}\text { N } 10.4 \pm 6.1 \\
\text { Monophonic W\% } 32.6 \pm 19.0 \% \\
\text { Polyphonic W\% } 53.6 \pm 25.5 \% \\
\text { Time without wheezes } 13.7 \pm 29.7 \%\end{array}$ \\
\hline $\begin{array}{l}\text { Taplidou } \\
\text { et al. } \\
\text { (2007) }\end{array}$ & $\begin{array}{l}\text { Cross- } \\
\text { sectional }\end{array}$ & $\begin{array}{l}7 \text { patients with } \\
\text { COPD } \\
\text { presenting } \\
\text { wheezes } \\
4 \mathrm{M}: 3 \mathrm{~F} \\
66.3 \pm 12.0 \mathrm{yrs} \\
\mathrm{FEV}{ }_{1} \\
54.5 \pm 18.2 \% \\
\text { predicted }\end{array}$ & $\begin{array}{l}\text { Respiratory sound recordings: } \\
\text { - semi-quiet clinical laboratory } \\
-5 \text { electret condenser microphones } \\
\text { (linear } \pm 1.5 \mathrm{~dB} \text { frequency response } \\
\text { of } 65-5,000 \mathrm{~Hz} \text { ) at trachea, right and } \\
\text { left axillae and right and left bases of } \\
\text { the posterior chest wall } \\
\text { - airflow of } 1.5 \mathrm{~L} / \mathrm{s}, \text { recorded with a } \\
\text { pneumotachograph }\end{array}$ & $\begin{array}{l}\text { Amplification and band-pass filter } 60- \\
2,100 \mathrm{~Hz} \\
\text { Sampling rate of } 5,512 \mathrm{~Hz} \\
\text { Wheeze detector based on time- } \\
\text { frequency analysis }\end{array}$ & $\begin{array}{l}\text { Wheezes: } \\
\text { N per recording }\end{array}$ & $\begin{array}{l}\text { Wheezes: } \\
\text { N 42 } \pm 30.6\end{array}$ \\
\hline
\end{tabular}




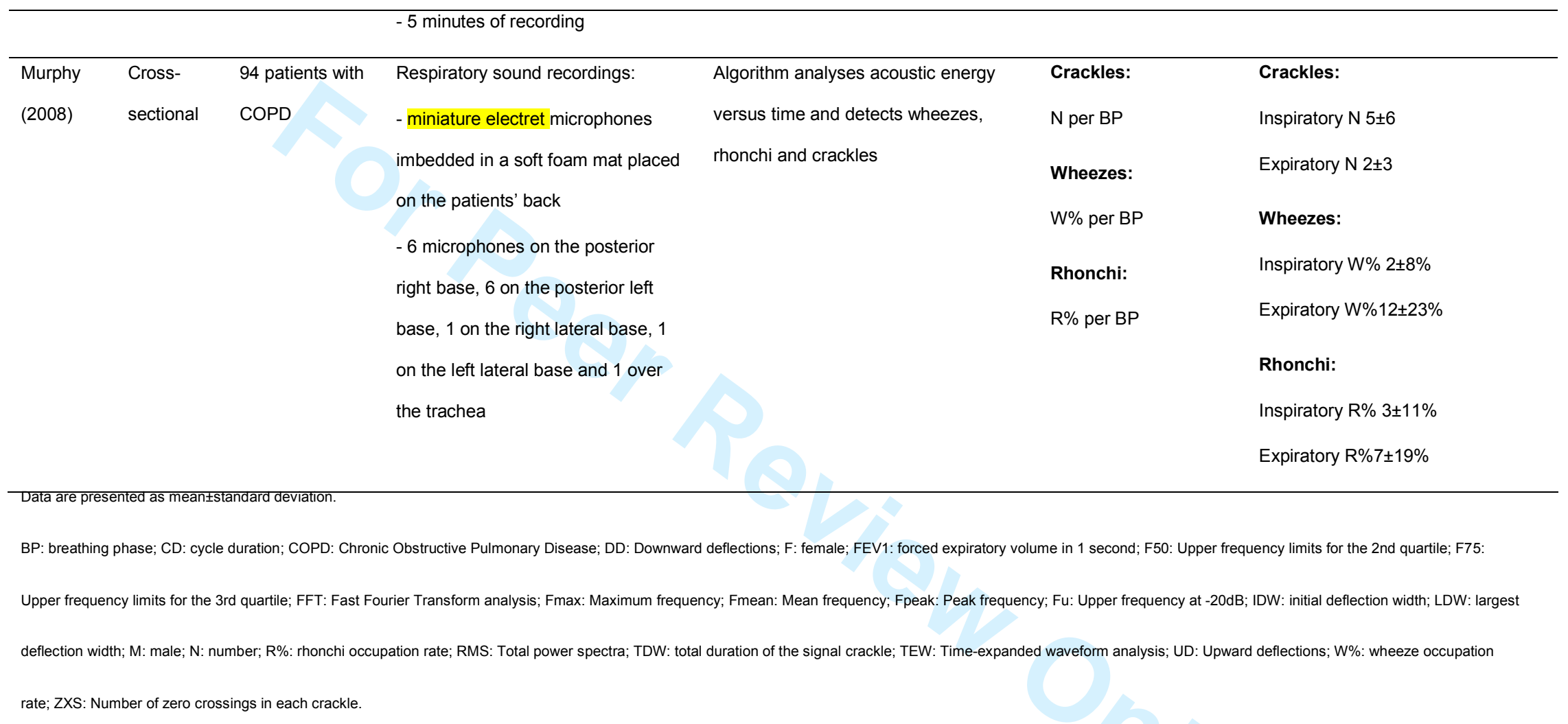




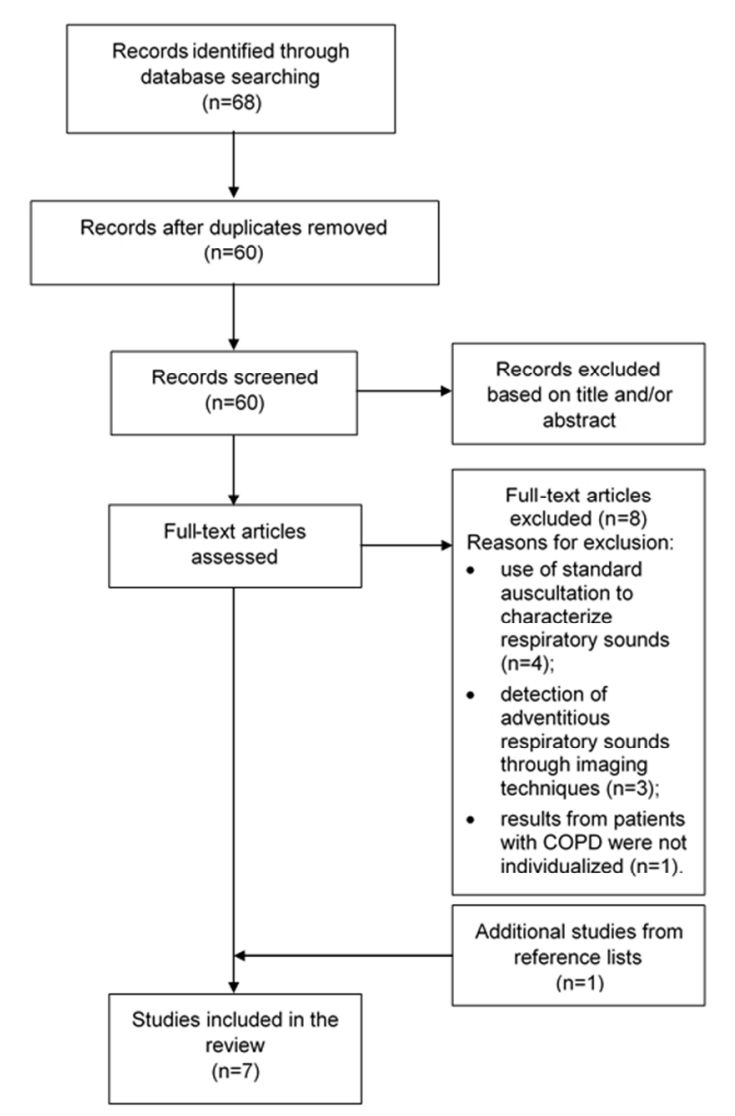

PRISMA flow diagram of the included studies. $245 \times 211 \mathrm{~mm}(96 \times 96 \mathrm{DPI})$ 


\section{Journal of COPD - Disclosure of Conflict of Interest}

Manuscript Title: Computerized respiratory sounds in patients with COPD: a systematic review

Corresponding Author: Alda Marques

A conflict of interest is defined as a set of circumstances or conditions in which one's judgment may be influenced by a secondary interest. The secondary interest is commonly financial gain; however, with respect to the Journal of COPD it would also include any interest that would influence or appear to influence one's professional judgment in the preparation of a manuscript for publication.

All authors are required to disclose all real or apparent conflicts of interest including financial relationships with any commercial entity that has an interest in the subject matter or materials discussed in the manuscript. (This includes the financial interests of one's spouse and children.) Potential sources of financial conflicts of interest include employment, consultancies, lecture fees, advisory boards, expert witness work, grants received or pending, patents received or pending, stock ownership or options, and royalties.

\begin{tabular}{|l|c|c|c|}
\hline \multicolumn{1}{|c|}{ Authors' Names } & $\begin{array}{c}\text { Conflict of } \\
\text { Interest }\end{array}$ & Signature & Date \\
\hline Cristina Jácome & No & Cistina Isabul Oliveira Jácome & $25 / 01 / 2014$ \\
\hline Alda Marques & No & Aldafofialiresde Dias Parquy & 25/01/2014 \\
\hline
\end{tabular}

Each author who identifies a real or potential conflict of interest must draft a statement of disclosure.

The corresponding author has responsibility to ensure completion of this form prior to editorial decision. Use additional sheets as necessary for disclosure statements.

Corresponding author:

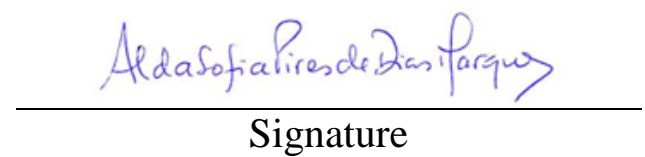

$25 / 01 / 2014$

Signature

Date 